\section{Imaging in Clinical Oncology}

A.D. Gouliamos, J.A. Andreou, and P.A. Kosmidis, eds.

New York, NY: Springer, 2014, 609 pages, \$229

The recent progress of oncologic imaging has been tremendous, and a multidisciplinary approach to each cancer patient is mandatory to ensure a longer and better life. Imaging specialists play a key role in providing information critical to making decisions about patient management. To achieve the highest standard of care, imaging specialists require not only detailed knowledge of the imaging findings but also an understanding of the current concepts of cancer development and growth and must be familiar with different staging classifications and treatment options. The pivotal role of imaging in monitoring and predicting therapeutic response must also be appreciated for timely detection of recurrent tumors and effective therapeutic interventions.

Medical imaging is an integral and indispensable part of the approach to cancer patients, and thus oncologists and imaging specialists should interact and comprehend each other's language. This book, edited by experienced radiologists and oncologists in Athens, Greece, highlights the benefits of radiologist-oncologist communication within the multidisciplinary team caring for cancer patients.

Well organized into 12 parts, the book includes 96 chapters covering various tumors. Each part begins by introducing a type of tumor and ends by discussing its clinical implications, along with the usefulness of combined imaging findings in its evaluation. All imaging techniques needed to prevent, diagnose, stage, evaluate therapeutic response, and follow up the particular tumor are described by expert imaging specialists. Part I, an introduction to oncologic imaging, consists of 5 chapters on molecular imaging in oncology, imaging criteria for therapeutic tumor response, imaging in radiation therapy, interventional radiology in oncology, and imaging in pediatric oncology. The 9 chapters of part II cover the use of conventional imaging, as well as ultrasound, CT, MR imaging, and PET, in soft-tissue and bone sarcomas, retroperitoneal tumors, and other bone and soft-tissue tumors. Part III, with 7 chapters, covers conventional and advanced MR imaging and PET as they relate to diagnostic issues with central nervous system tumors. Part IV is on lung cancer and has 6 chapters addressing cancer screening in at-risk patients using low-dose CT, CT-MR imaging, PET, and endobronchial ultrasound. Part V discusses head and neck cancer, with 5 chapters reviewing ultrasound, CT, $\mathrm{MR}$, and PET/CT findings. Part VI contains 3 chapters dealing with CT, MR imaging, and PET/CT in the staging of lymphomas. Part VII discusses gynecologic cancer, with 8 chapters about ultrasound, CT-MR imaging, and PET/CT findings in cervical, endometrial, and ovarian cancers. Part VIII covers breast cancer, with 6 chapters on x-ray and MR mammography and on PET/ CT. Part IX, with 16 chapters, addresses imaging findings for gastrointestinal cancer, including tumors of the esophagus, stomach, liver, pancreas, peritoneal cavity, and large bowel. Part X, with 3 chapters, reviews neuroendocrine tumors. Part XI deals with urogenital cancer, including imaging of adrenal, renal, urothelial, testicular, and prostate tumors with ultrasound, CT, MR imaging, and PET/CT. Finally, part XII has 3 chapters reviewing the imaging findings of melanoma.

The book is well written and conveys the information clearly. The figures and tables are of high quality, as is the paper on which they are printed. The references in each chapter are current, and the index is helpful.

I am sure this book will serve as a guide through which imaging specialists and clinicians, especially oncologists, can rapidly acquire the basic knowledge essential to daily oncologic practice. I highly recommend this book to trainees and practitioners in oncology, radiology, nuclear medicine, internal medicine, and family medicine.

\author{
E. Edmund Kim \\ University of California at Irvine \\ 101 The City Dr. S. \\ Orange, CA 92868 \\ E-mail: edmundek@uci.edu
}

